\title{
MS03-03 | In Numbers: Initial Screening at the MRC-LMB Protein Crystallization
}

\section{FACILITY}

Gorrec, Fabrice (MRC Laboratory of Molecular Biology, Cambridge, GBR)

A strategy to bypass some difficulties related to protein crystallisation is to use a large initial screen containing many different initial crystallization conditions. We have adopted such a strategy at the MRC Laboratory of Molecular Biology (MRC-LMB, Cambridge, UK), combined with an automated vapour-diffusion sitting drop technique for setting up crystallization droplets. To further simplify the process when dealing with large screens, crystallization plates pre-filled with reservoir solutions are made ready to be used. Here, I present some statistics collected over a decade of running the facility at MRC-LMB: the numbers of plates pre-filled with initial screening kits, the resulting costs and the final yields of quality diffraction crystals. The numbers also highlight a recent decline in pre-filled plates consumption caused by the advent of a competing method that eliminates the need for crystallization - cryo-electron microscopy (cryo-EM) -, which now regularly produces atomic models comparable in quality to those obtained by X-ray crystallography. 